CONGENITAL HEART DISEASE

\title{
Sinus venosus syndrome: atrial septal defect or anomalous venous connection? A multiplane transoesophageal approach
}

\author{
J M Oliver, P Gallego, A Gonzalez, F J Dominguez, A Aroca, J M Mesa
}

\begin{abstract}
Objective: To discuss the anatomical features of sinus venosus atrial defect on the basis of a comprehensive transoesophageal echocardiography (TOE) examination and its relation to surgical data. Methods: 24 patients (13 men, 11 women, mean (SD) age 37 (17) years, range 17-73 years) with a posterior interatrial communication closely related to the entrance of the superior (SVC) or inferior vena cava (IVC) who underwent TOE before surgical repair. Records of these patients were retrospectively reviewed and compared with surgical assessments.

Results: In 13 patients, TOE showed a deficiency in the extraseptal wall that normally separates the left atrium and right upper pulmonary vein from the SVC and right atrium. This deficiency unroofed the right upper pulmonary vein, compelling it to drain into the SVC, which overrode the intact atrial septum. In three patients, TOE examination showed a defect in the wall of the IVC, which continued directly into the posterior border of the left atrium. Thus, the intact muscular border of the atrial septum was overridden by the mouth of the IVC, which presented a biatrial connection. In the remaining eight patients, the defect was located in the muscular posterior border of the fossa ovalis. A residuum of atrial septum was visualised in the superior margin of the defect. Neither caval vein overriding nor anomalous pulmonary vein drainage was present.

Conclusions: Sinus venosus syndrome should be regarded as an anomalous venous connection with an interatrial communication outside the confines of the atrial septum, in the unfolding wall that normally separates the left atrium from either caval vein. It results in overriding of the caval veins across the intact atrial septum and partial pulmonary vein anomalous drainage. It should be differentiated from posterior atrial septal defect without overriding or anomalous venous connections.
\end{abstract}

\begin{abstract}
A though Peacock in $1858^{1}$ and Waggstaffe in $1868^{2}$ described the pathology of sinus venosus atrial defect(SVD), the anatomical criteria for diagnosis and the embryology of this infrequent cardiac disorder are still contentious. $^{3-7}$ Several morphological and echocardiographic studies $^{6-10}$ have already suggested that the pathognomonic diagnostic feature is an extraseptal interatrial communication with an anomalous caval vein connection with or without an associated anomalous pulmonary venous drainage. However, the nature of this deficiency has not been established, as accurate evaluation of normal atrial septal anatomy and a precise definition of the septal borders remain matters of concern.

Preoperative diagnosis is also a challenge and has implications for surgical planning. Previously reported transoesophageal echocardiographic (TOE) findings of SVD have failed to recognise the pathognomonic morphological features of SVD. ${ }^{11}$ As the detection of SVD by the transthoracic echocardiographic approach in adults has several limitations ${ }^{12}$ (the posterior location of the defect makes it difficult to identify from precordial examination), there is no doubt that TOE should be the procedure of choice for the diagnosis of SVD $\mathrm{SV}^{13-16}$ and the associated congenital anomalies. ${ }^{17}$ Therefore, the aim of this report is to discuss the anatomical features of SVD on the basis of preoperative TOE examination and its relation to surgical assessment.
\end{abstract}

\section{PATIENTS AND METHODS}

Data were collected from the database of the Adult Congenital Heart Disease Unit from January 1990 to December 2000 at La Paz Hospital, Madrid. The study population comprised all patients over 15 years of age with a posterior interatrial communication closely related to the entrance of the superior
(SVC) or inferior vena cava (IVC) who underwent TOE before surgical repair. We also determined the number of adults with atrial septal defect or congenital heart disease diagnosed by echocardiographic or surgical examination during the same period.

TOE examination was performed preoperatively as an outpatient procedure in all the cases using a Sonos 1500, 2500, or 5500 ultrasound system (Philips, Andover, Massachusetts, USA) fitted with a 5.0/3.5 MHz multiple frequencies multiplane transducer. The atrial septum was imaged in the four chamber, basal short axis, longitudinal vena cava, and intermediate views. Drainage and the connection of the pulmonary and caval veins was visualised by two dimensional imaging combined with colour flow Doppler. Agitated saline contrast echocardiography and colour flow Doppler imaging were used to detect any bidirectional shunting between the left an right atria.

Records of the TOE examination of each patient in which an SVD had been diagnosed preoperatively were reviewed retrospectively in light of the surgical anatomical descriptions. Echocardiographic and surgical observations were then compared to address the anatomical features of SVD and its correspondence to the echocardiographic images.

\section{RESULTS}

From 1990 to December 2000, congenital heart disease was diagnosed in 2165 patients older than 15 years. Atrial septal

Abbreviations: IVC, inferior vena cava; RUPV, right upper pulmonary vein; SVC, superior vena cava; SVD, sinus venosus atrial defect; $T O E$, transoesophageal echocardiography 

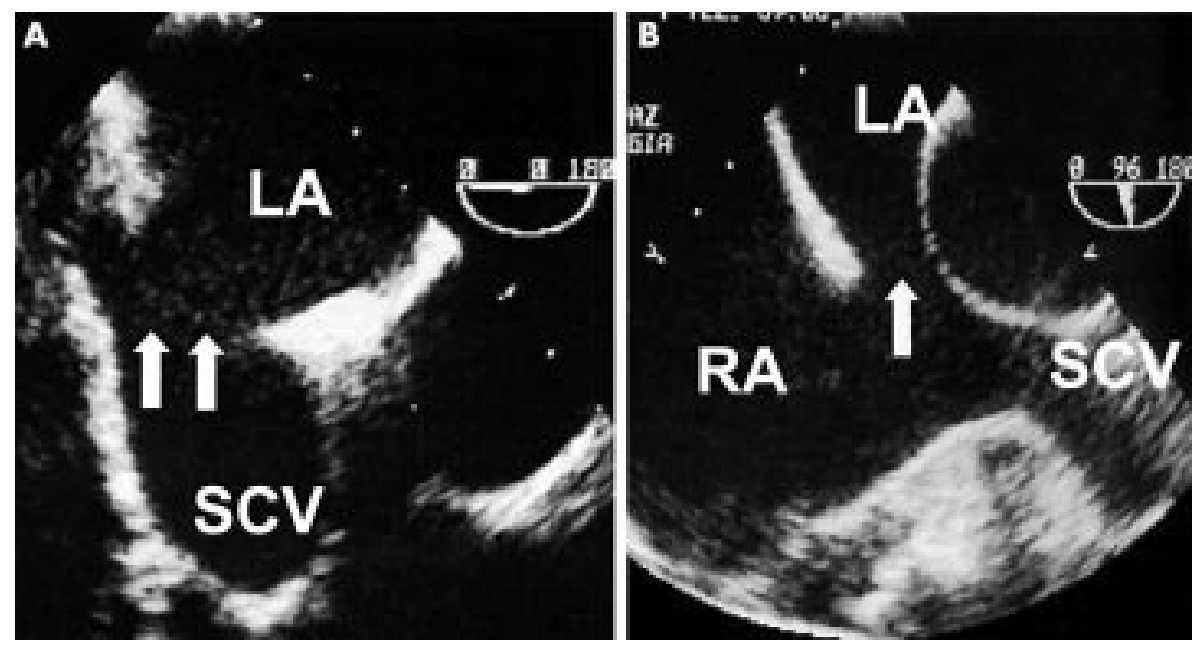

Figure 1 (A) A deficiency in the wall that normally separates the left atrium (LA) and right upper pulmonary vein from the superior vena cava (SCV) in the short axis view. This deficiency unroofed the right upper pulmonary vein, compelling it to drain into the superior vena cava or into the superior dome of the right atrium (RA) at the level of the caval atrial junction. (B) From the long axis view, the defect that connected the left and right atria was located outside the confines of the atrial septum, just inferior to the right pulmonary artery. No atrial septal tissue at the superior or posterior margin of the defect was imaged. Overriding of the superior vena cava across the intact atrial septum with a biatrial connection was seen.

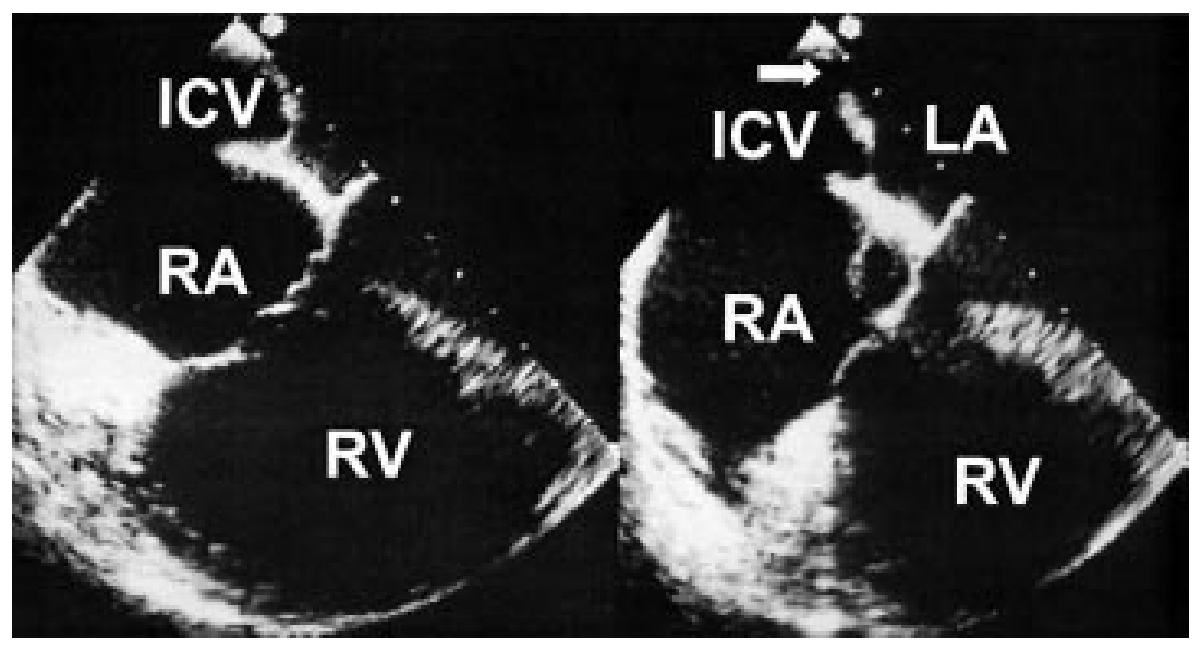

Figure 2 Transoesophageal echocardiography shows a defect in the wall of the inferior vena cava (ICV) that continues directly into the inferoposterior border of the interatrial septum. The fossa ovalis of the atrial septum was overridden by the mouth of the inferior cava, which also had a biatrial connection. No residuum of atrial septal tissue was present at the inferior margin of the defect. $L A$, left atrium; RA, right atrium $R V$, right ventricle.

defect was diagnosed echocardiographically or surgically in 332 (15\%) and SVD in 41, bringing the prevalence of SVD in the group of adults with congenital heart disease to $1.2 \%$ and in the group with atrial septal defect to $12 \%$. Twenty four patients with SVD who had a preoperative TOE examination made up the study population. Mean (SD) age was 37 (17) years (range 17-73). There were 13 men and 11 women.

TOE records were reinterpreted based on the surgical notes. At surgery, SVC or IVC overriding across the atrial septum was found in 16 patients. A deficiency in the extraseptal unfolding that normally separates the left atrium from SVC or IVC was found in those patients. Partial anomalous right upper pulmonary vein (RUPV) connection into the SVC or the superior dome of the right atrium at the level of the caval atrial junction was present in 13 of these patients. In the other eight patients the defects were reclassified at surgery as posterior atrial septal defects located inside the confines of the atrial septum. No right sided anomalous pulmonary or systemic venous drainage was identified at the time of surgery in these eight patients. Normal left sided pulmonary venous drainage was confirmed in all 24 patients.

According to a retrospective review of TOE, the population was divided into three groups. Group 1 consisted of 13 patients who had a deficiency in the wall that normally separates the left atrium and RUPV from the SVC in the short axis view (fig lA). This deficiency unroofed the RUPV, compelling it to drain into the SVC or into the superior dome of the right atrium at the level of the caval atrial junction. An anomalous connection of the RUPV entering the SVC gave the SVC a teardrop appearance. From the long axis view, the defect that connected the left and right atria was located outside the confines of the atrial septum, just inferior to the right pulmonary artery. The fossa ovalis and superior muscular border of the atrial septum were actually intact and no atrial septal tissue at the superior or posterior margin of the defect was seen. Overriding of the SVC across the intact atrial septum with biatrial connection was shown in every case from the caval vein long axis view (fig lB).

Group 2 consisted of three patients in whom TOE examination showed a defect in the wall of the IVC that continued directly into the inferoposterior border of the left atrium (fig 2 ). The fossa ovalis of the atrial septum was overridden by the mouth of the IVC, which also had biatrial connection. No residuum of atrial septal tissue was present at the inferior margin of the defect. A normal pulmonary venous connection was imaged in these three cases.

Group 3 consisted of eight patients in whom the defect was located in the muscular posterior and superior border of the fossa ovalis. A residuum of atrial septum was visualised in the superior margin of the defect in both the short and the long axis view of the SVC (fig 3 ). Neither caval vein overriding nor anomalous pulmonary vein connection was identified in these eight patients.

\section{DISCUSSION}

\section{Anatomy of atrial septum}

Previously published morphological studies ${ }^{3-10}$ have sought to re-establish the anatomy of the SVD based on comprehensive analysis of normal atrial septal structures. Characterisation of the atrial septum would aid in understanding the morphology of the communications that can exist between the atrial 


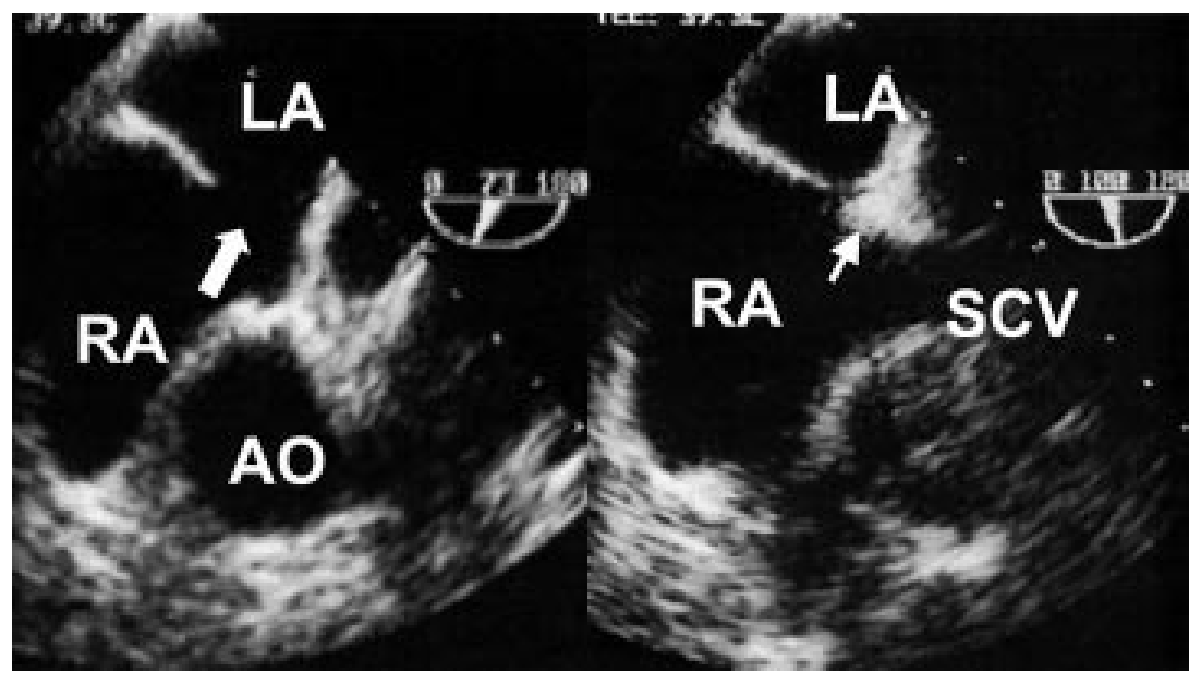

Figure 3 Short and long axis views of the superior vena cava (SCV) showing a defect located in the muscular posterior and superior border of the fossa ovalis. A residuum of atrial septum was visualised in the superior margin of the defect. Neither caval vein overriding nor anomalous pulmonary vein connection was identified. AO, aorta; LA, left atrium; $R A$, right atrium. chambers, not all of which are true septal defects. Anderson and colleagues ${ }^{4}$ and $\mathrm{Al}$ Zaghal and associates ${ }^{6}$ defined a true septal structure as one that can be removed without exiting the cavities of the heart. It is the flap valve of the fossa ovalis, along with its muscular rim, that fulfils this criterion. The remainder of the posterior atrial septum is no more than an unfolding between the walls of the right and left atria and the drainage of systemic and pulmonary venous, incorporating a core of extracardiac fibroadipose tissue. True defects of the atrial septum therefore exist because of deficiency, perforation, or absence of the flap valve or muscular rim of the fossa ovalis. The other interatrial communications are outside the confines of the atrial septum. Recognition of this feature is the key to SVD diagnosis and to its differentiation from true atrial septal defects. ${ }^{8-10}$

\section{Overriding of caval veins}

Al Zaghal and colleagues ${ }^{6}$ reported that the pathognomonic feature of SVD is the overriding of the intact rim of the fossa ovalis by the SVC or IVC and the presence of a shunt at the atria level. Although a defect within the true atrial septum can coexist with an SVD, the most reliable and unequivocal diagnosis is made by confirming that the muscular border of the fossa ovalis is intact. Overriding of caval veins was shown by TOE and was confirmed at the surgical examination in 16 patients in this series (groups 1 and 2). In all of these cases, atrial communication was located outside the true septal structures. The deficiency was located in the wall that normally separates the left atrium from the SVC (group 1) or the IVC (group 2) and there was no atrial septal tissue at the posterior margin of the defect. In the other eight misdiagnosed patients, a residuum of atrial septum was visualised beneath the entry of both caval veins into the right atrium, but overriding across the atrial septum of caval veins was not found at TOE or surgery. Thus, patients in group 3 should be reclassified as having an ostium secundum type of atrial septal defect with a posterior defect inside the confines of the true atrial septum. Only patients of groups 1 and 2 fulfil the anatomical criteria of SVD-that is, overriding of SVC or IVC across the atrial septum and no residuum of atrial septum beneath the biatrial connection of the SVC or IVC.

\section{Unroofing of pulmonary veins}

SVD is frequently associated with a right sided anomalous pulmonary venous connection. Up to $85 \%$ of patients with a superior SVD have anomalous right pulmonary venous drainage. ${ }^{18}$ In fact, the most common type of partial anomalous pulmonary venous connection is an RUPV to an SVC or to the superior caval atrial junction, as it corresponds to the anomalous connection associated with SVD, reported to occur in up to $49 \%$ of echocardiographic studies of patients with partial anomalous pulmonary venous connection. ${ }^{17}$ Van Praagh and colleagues ${ }^{3}$ reported that SVD appears to be a deficiency in the wall that normally separates the right pulmonary veins from the right sinus venosus. This deficiency unroofs the pulmonary vein, permitting it to drain into the right side of the heart. Unroofed RUPV was shown by TOE in all patients of group 1 in this series, but it was not found at TOE or surgical examination in groups 2 or 3 . The TOE basal short axis view at the SVC level in normal subjects shows that the wall separating the SVC from the left atrium is formed by the roof of the RUPV entering the left atrium. A deficiency in this wall unroofs the RUPV, resulting not only in overriding across the atrial septum of the SVC but also in anomalous connection of the RUPV. A partial anomalous RUPV connection to either the SVC or the superior caval atrial junction was shown by colour flow Doppler at TOE examination in all patients of group 1. Echocardiographic findings were confirmed by surgical inspection in each case.

Anomalous pulmonary venous connection was not found at TOE or surgical examination in patients of group 2. In fact, the wall separating the IVC from the left atrium does not form a roof over the pulmonary veins in normal subjects. Thus, a deficiency in this extraseptal wall results in overriding of the IVC but not in the anomalous pulmonary vein connection. The most frequent partial anomalous pulmonary vein connection to the IVC is the scimitar syndrome, but an interatrial communication is not usually found in adult patients with this syndrome. ${ }^{19}$ If Van Praagh's criterion of right pulmonary vein unroofing is necessary for the diagnosis of SVD, most IVC-type atrial septal defects should not be classified into this group. However, we agree with other authors ${ }^{46-10}$ in considering the overriding of the intact rim of the fossa ovalis by the SVC or IVC to be the pathognomonic feature of SVD. Thus, an IVC type of SVD should be the diagnosis in group 2 patients.

\section{Prevalence of SVD}

The prevalence of SVD varies from $2-10 \%$ of patients with atrial septal defect. ${ }^{20}{ }^{21}$ When all patients with an echocardiographic diagnosis of SVD were considered, the prevalence in this study was estimated at $12 \%$ of the adults with an atrial septal defect. This relatively high prevalence may be explained by a selection bias, since our patients were drawn from the database of an adult congenital heart disease clinic, to which only patients with complex forms of atrial septal defect were referred. However, this prevalence may also indicate overdiagnosis of SVD in light of the current echocardiographic criteria: a defect in the posterior region of the atrial septum, closely related to the entrance of the SVC or the IVC, with minimal or no septal tissue separating the defect from the posterior atrial 


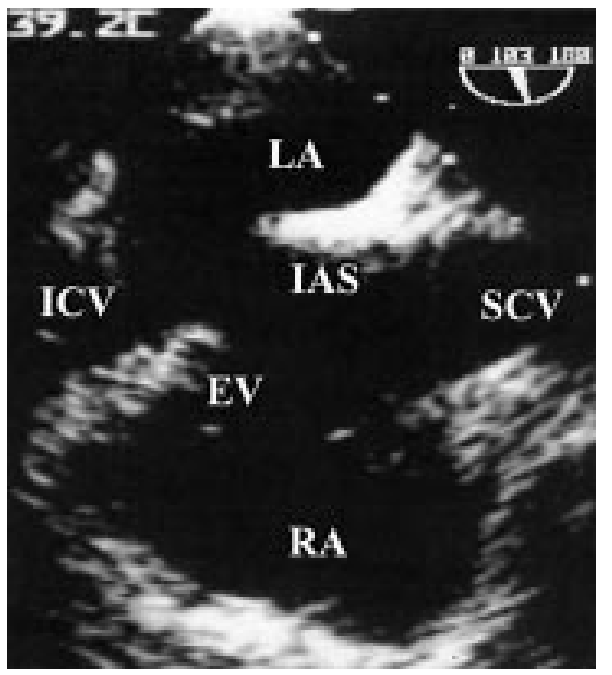

Figure 4 Transoesophageal echocardiographic caval vein long axis view showing inferior sinus venosus. The posterior wall of the inferior vena cava (ICV) continues into the posterior border of the left atrium (LA). At surgery, a large eustachian valve (EV) may be mistaken for the lower margin of the defect. IAS, interatrial septum; $\mathrm{RA}$, right atrium; SCV, superior vena cava.

wall. ${ }^{12}{ }^{22}$ More restrictive echocardiographic criteria for diagnosis should probably be established.

\section{Transoesophageal echocardiography}

TOE is considered to be the procedure of choice to diagnose $\mathrm{SVD}^{7}$ and its associated anomalies ${ }^{9}$ in adults. Although longitudinal planes from the mid-oesophageal position are the most reliable views, the atrial septal anatomy and venous connections are more readily examined with multiplane transducers, which allow multiple short axis, intermediate, and longitudinal scan images of the defect to be obtained. Previous reports showing the TOE features of SVD have failed to recognise and even to discuss the main morphological features of $\mathrm{SVD}^{11}$ : the extraseptal nature of the atrial communication, overriding of the caval vein, and the unroofed RUPV. Recognition of these anatomical hallmarks is essential not only in differentiating true SVD from the posterior location of secundum-type atrial septal defects but also in making the surgical approach. This retrospective review of TOE examinations intends to show that, with proper technique, a reliable diagnosis of SVD is possible when the intact fossa ovalis overridden by the mouth of the SVC or the IVC is seen. Thus, the absence of atrial septal tissue at the superior or posterior margin of the defect on a longitudinal scan at the level of the caval atrial junction and the overriding of the SVC or the IVC should be considered the key to diagnosis. Furthermore, in our own experience, preoperative identification of SVD and right sided anomalous venous connection and its differentiation from a posterior atrial septal defect have expanded the role of TOE in anatomical discussion of atrial septal communications. Nevertheless, a false positive diagnosis may be made when a posterior septal defect within the confines of the fossa ovalis is associated with an anomalous venous drainage.

\section{Surgical implications}

Surgical repair of the SVD has been associated with postoperative sinus node dysfunction, ${ }^{23}{ }^{24}$ venous obstruction, ${ }^{25}$ and right to left shunting. ${ }^{27}{ }^{28}$ Preoperative recognition of the extraseptal nature of the interatrial communication may avoid surgical damage to the sinus node by using a transcaval approach for repair. ${ }^{29}$ Obstruction of the SVC or right pulmonary veins after surgery can also be avoided by finding biatrial SVC connection and anomalous pulmonary vein connection before planning surgical procedure. Following surgical repair of an inferior SVD, residual right to left shunt has been reported when the lower portion of the defect is not closed because a biatrial connection of the IVC is not seen. Occasionally, the eustachian valve of the IVC has been mistaken for the lower margin of the defect and blood flow was deferred from the IVC to the left atrium ${ }^{67} 2830$ (fig 4). Correct recognition of the anatomy of the SVD and intraoperative TOE guided intervention may avoid these surgical mistakes.

\section{Conclusions}

Sinus venosus syndrome should be regarded as an anomalous venous connection with an anatomical interatrial communication outside the confines of the fossa ovalis, in the unfolding wall that normally separates the left atrium and the SVC or IVC. It results in overriding of the SVC and IVC across the intact atrial septum and partial anomalous pulmonary drainage when the RUPV is unroofed by the defect. It should be differentiated from posterior atrial septal defects without overriding or anomalous venous connections. TOE is a very accurate tool for diagnosis, surgical planning, and intraoperative monitoring of this uncommon disorder.

\section{Authors' affiliations}

J M Oliver, A Gonzalez, F J Dominguez, A Aroca, J M Mesa, Adult

Congenital Heart Disease Unit, La Paz Hospital, Madrid, Spain

P Gallego, Virgen Macarena Hospital, Sevilla, Spain

\section{REFERENCES}

1 Peacock TB. Malformations dependent on arrest of development at an early period of fetal life. In: Peacock TB, ed. Malformations of the human heart. London: Churchill, 1858:24-5.

2 Waggstaffe WW. Two cases of free communication between the auricles, by deficiency of the upper part of the septum auricularum. Trans Pathol Soc Lond 1868;19:91-8.

3 Van Praagh S, Carrera ME, Sanders SP, et al. Sinus venosus defects: unroofing of the right pulmonary veins-anatomic and echocardiographic findings and surgical treatment. Am Heart J 1994;1 28:365-79.

4 Anderson RH, Ettedgui JA, Devine WA. Sinus venous defect. Am HeartJ 1995; 129:1229-30.

5 Van Praagh S, Carrera ME, Sanders SP, et al. Sinus venosus defect [reply]. Am Heart J 1995;129:1231-2.

6 Al Zaghal, Ahmed M, Li J, et al. Anatomical criteria for diagnosis of sinus venosus syndrome. Heart 1997;78:298-304

7 Blom NA, Gittenberger-de Groot AC, Jongeneel TH, et al. Normal development of the pulmonary veins in human embryos and formulation of a morphogenetic concept for sinus venosus defects. Am J Cardiol 2001;87:305-9.

8 Ettedgui JA, Siewerds RD, Anderson RH, et al. Diagnostic echocardiographic features of the sinus venosus defect. Br Heart J 1990;64:329.

9 Li J, Al Zaghai AM, Anderson RH. The nature of the superior sinus venosus defect. Clin Anat 1998;11:349-52

10 Ferreira Martins JD, Anderson RH. The anatomy of interatrial communications: what does the interventionist need to know? Cardiol Young 2000;10:464-73.

11 Pascoe RD, Oh JK, Warnes CA, et al. Diagnosis of sinus venosus atrial septal defect with transesophageal echocardiography. Circulation 1996;94:1049-55

12 Nasser FN, Tajik AJ, Seward JB, et al. Diagnosis of sinus venosus atrial septal defect by two-dimensional echocardiography. Mayo Clin Proc 1981;56:568-72

13 Oh JK, Seward JB, Khandheria BK, et al. Visualization of sinus venosus atrial septal defect by transesophageal echocardiography. J Am Soc Echocardiogr 1988;1:275-7.

14 Kronzon I, Tunick PA, Freedberg RS, et al. Transesophageal echocardiography is superior to transthoracic echocardiography in the diagnosis of sinus venosus atrial defect. J Am Coll Cardiol 1991;17:537-42.

15 Ricou FJ, Reynard CA, Lerch R. Transesophageal echocardiography in the diagnosis of inferior caval secundum atrial septal defect. Am Heart J 1994; 128:196-9

16 Watanabe F, Takenaka K, Suzuki J, et al. Visualization of sinus venosus-type atrial septal defect by biplane transesophageal echocardiography. J Am Soc Echocardiogr 1994;7:179-81.

17 Ammansh NM, Seward JB, Warnes CA, et al. Partial anomalous venous connection: diagnosis by transesophageal echocardiography. J Am Coll Cardiol 1997;29:1351-8

18 Gustafson RA, Warden HE, Murray GF, et al. Partial anomalous pulmonary venous connection to the right side of the heart. J Thorac Cardiovasc Surg 1989;98:861-8.

19 Dupuis C, Charaf LA, Breviere GM, et al. The "adult" form of the scimitar syndrome. Am J Cardiol 1992;70:502-7. 
20 Swan HJC, Kirklin JW, Becu LM, et al. Anomalous connnection of right pulmonary veins to superior vena cava with interatrial communications: hemodynamic data in eight cases. Circulation 1957; 16:54-65.

21 Davia JE, Cheitlin MD, Bedynek JL. Sinus venosus atrial septal defect. Am Heart J 1973:85: 177-85.

22 Snider AR, Serwer GA. Echocardiography in pediatric heart disease. Chicago: Year Book Medical Publishers, 1990:134-67.

23 Kyger ER, Frazier OH, Cooley DA, et al. Sinus venosus atrial septa defect: early and late results following closure in 109 patients. Ann Thorac Surg 1978:25:44-50.

24 Trusler GA, Kazenelson G, Freedom RM, et al. Late results following repair of partial anomalous pulmonary venous connection with sinus venosus atrial septal defect. J Thorac Cardiovasc Surg 1980;79:776-81.

25 Weber HS, Markowitz RI, Hellenbrand WE, et al. Pulmonary venous collaterals secondary to superior vena cava stenosis: a rare cause of right-to-left shunting following repair of a sinus venosus atrial septal defect. Pediatr Cardiol 1989;10:49-51
26 DeLeon SY, Freeman JE, Ilbawi MN, et al. Surgical techniques in partia anomalous pulmonary veins to the superior vena cava. Ann Thorac Surg 1993:55:1222-6.

27 Desnick SJ, Neal WA, Nicoloff DM, et al. Residual right-to-left shunt following repair of atrial septal defect. Ann Thorac Surg 1976;21:291-5.

28 Sapin PM, Salley RK. Arterial desaturation and orthodeoxia after atrial septal defect repair: demonstration of the mechanism by transesophagea and contrast echocardiography. J Am Soc Echocardiogr 1997; 10:588-92

29 Nicholson IA, Chard RB, Nunn GR, et al. Transcaval repair of the sinus venosus syndrome. J Thorac Cardiovasc Surg 2000;1 19:741-4.

30 Becker A, Buss M, Sebening W, et al. Acute inferior cardiac inflow obstruction resulting from inadvertent surgical closure of a prominent Eustachian valve mistaken for an atrial septal defect. Pediatr Cardio 1999;20: 155-7.

\section{IMAGES IN CARDIOLOGY}

\section{Pronounced PR depression}

A 43 year old man with constrictive pericarditis underwent pericardiectomy with good results. Surgical pathology showed thickened pericardium with mild non-specific acute and chronic inflammation, and no granulomas. On postoperative day 2, he was asymptomatic and haemodynamically stable, but was noted to have pronounced PR depression on ECG in comparison with baseline (upper panel). Drainage of $135 \mathrm{ml}$ of mediastinal fluid occurred and normalisation of the PR segment was noted instantaneously (lower panel). Repeated physical examination on subsequent days revealed friction rub. The patient was treated with naproxen and was eventually discharged from hospital. The most likely explanation for the dynamic PR segment change is transient accumulation of mediastinal fluid around the heart in the setting of residual inflammation postpericardiectomy.

C Lee

W Czarnecki wczarnecki@manitobaclinic.com
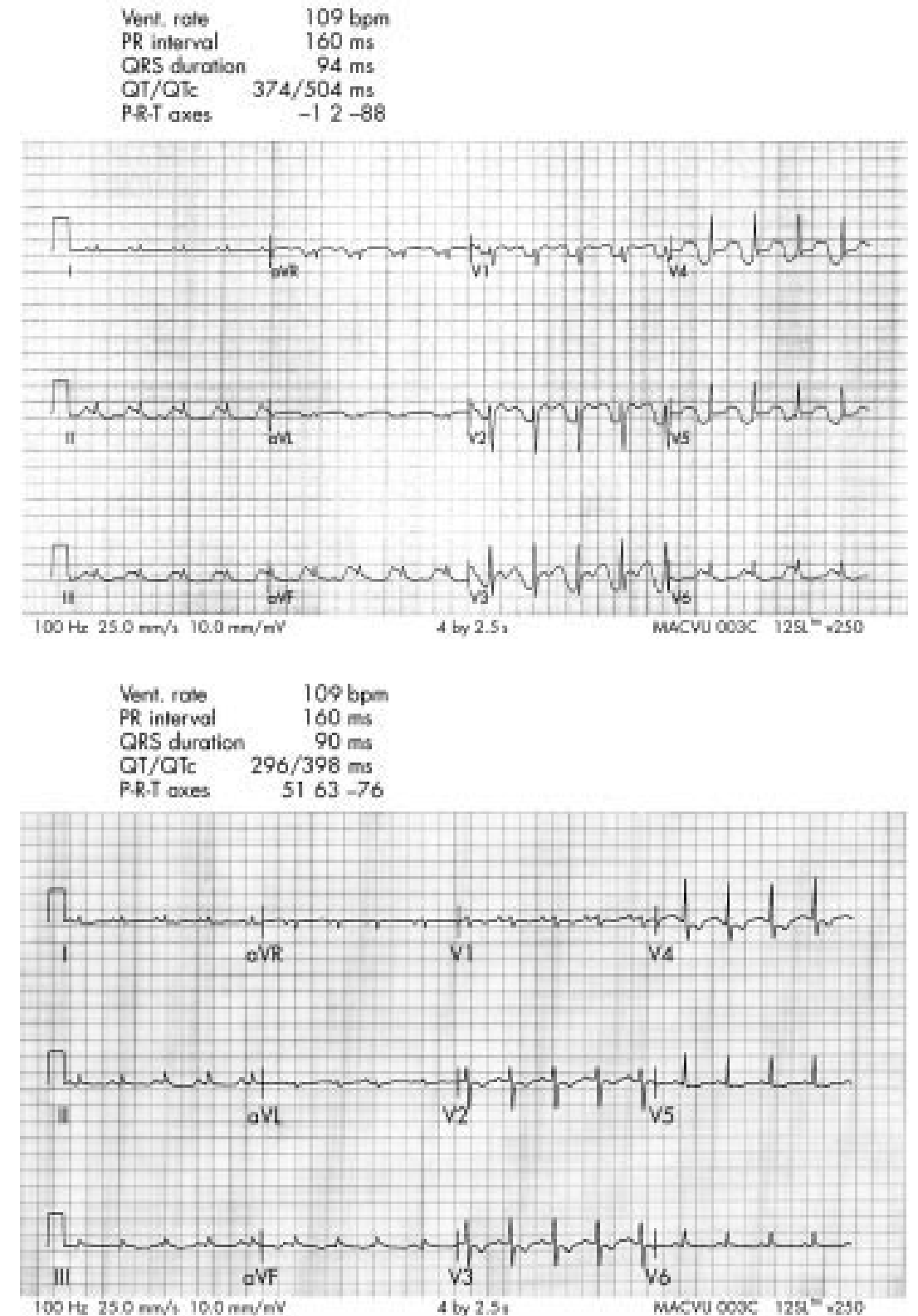